\title{
A New Method for Numerical Calculation of Pressure Gradient of Unsteady Flow of a Viscoelastic Fluid in Eccentric Annulus
}

\author{
Li Nan \\ Production Engineering and Research Institute of Daqing Oilfiled Co. Ltd., Daqing, China \\ linanln2000@163.com
}

\begin{abstract}
Finite Volume Method (FVM) was used to discrete governing equations of unsteady flow of secondorder fluid with variable coefficients in eccentric annulus with the inner cylinder reciprocating axially, in which a fully implicit scheme was taken on time integral, and Alternating Direction Implicit (ADI) method to solve the discrete equation group. Using HPAM aqueous solution, the numerically calculated pressure gradients were compared with the experimentally measured ones, and coincidences of the comparisons were compared with those calculated by using the other two old methods. The results show that the new method presented is testified to be correct and the coincidences between the calculated pressure gradients and the measured ones, using the new method, are better than those using the old methods; moreover, the numerical calculating procedure of the new method is much more simple and convenient and calculating speed is faster. This study is of certain significance in practical engineering for optimizing the working parameters of beam pumping unit and the energy saving of production system of the unit.
\end{abstract}

Keywords-Pressure Gradient; Unsteady Flow; Viscoelastic Fluid; Eccentric Annulus; Numerical Calculation

\section{INTRODUCTION}

In petroleum engineering, during the oil production of polymer-flooding, flow of the pumping fluid in wellbore can be regarded as unsteady flow of the fluid in eccentric annulus with the inner cylinder reciprocating axially. A viscoelastic model of second-order fluid with variable coefficients, presented by Noriyasu ${ }^{[1,2]}$ et al, is used to describe the rheology of the pumping fluid. On the unsteady flow of the second-order fluid in eccentric annulus with the inner cylinder reciprocating axially, Yang $^{[3,4]}$ et al presented the velocity distribution and the pressure distribution on the wall of the inner cylinder, in which Finite Difference Method (FDM) was used to discrete the governing equations with semi-implicit CrankNicholson (C-N) scheme taken on time integral and the discrete equation group was numerically solved by GuassSeidel method, which can be called FDM-CN-GS method here for short; Zhang ${ }^{[5-8]}$ et al presented the distribution of the instantaneous flow rates and numerically calculated the average flow rates, then, gave an experimental verification, in which Finite Volume Method (FVM) was used to discrete the governing equations with semi-implicit $\mathrm{C}-\mathrm{N}$ scheme taken on time integral and the discrete equation group was numerically solved by Successive Over Relaxation (SOR) method, which can be called FVM-CN-
SOR method here for short, and it was compared with FDM-CN-GS method, the result showed that under the same conditions, the solutions numerically obtained by using these two methods coincided well with each other, but to get the same accuracy requirement, the discretion steps were more simple and convenient, the computation speed was faster if using FVM-CN-SOR method.

However, Yang ${ }^{[9,10]}$ et al pointed out that C-N scheme was usually described as an unconditional stable one, but it did not mean that no matter how large the time step was the physical real solution could be obtained. This was because the mathematical stability could only guarantee that the oscillation during the process of iteration solving would vanish, namely, converge, but not that the physical real solution could be obtained, whereas, the Fully Implicit (FI) scheme could guarantee that both of the mathematical convergence requirement and physical real solution could be obtained.

Therefore, here FVM is used to discrete the governing equations with FI scheme taken on time integral instead, and Alternating Direction Implicit (ADI) method to numerically solve the discrete equation group, which can be called FVM-FI-ADI method here for short. Using Hydrolyzed Polyacrylamide (HPAM) aqueous solution, the mass fraction concentration of which was $0.1 \%$, regarded as the second-order fluid with variable coefficients, under the same conditions, on the basis of using FVM-FI-ADI method to numerically solve the governing equations and obtain the velocity field, connected with pressure gradient equation, through comparing the numerically calculated pressure gradients with experimentally measured ones, the new method is testified to be correct through experiment, and compare the coincidence with that by using FDM-CN-GS and FVMCN-SOR, respectively. This study is of certain significance in practical engineering for optimizing the working parameters of beam pumping unit and the energy saving of production system of the unit.

\section{GOVERNING EQUATIONS}

\section{A. Hypothetical conditions}

Consider the isothermal unsteady laminar flow of the isotropic incompressible second-order fluid with variable coefficients in an infinitely long vertical eccentric annulus, with the inner cylinder reciprocating axially at a velocity of $U(t)=\pi S f \sin 2 \pi f t$ in a period of $T=1 / f$, where the stroke and the stroke frequency of the inner cylinder are 
$S$ and $f$, respectively, and that the radiuses of the inner and the outer cylinder are $R_{\text {in }}$ and $R_{\text {out }}$, respectively, the annular eccentricity is $e p$, the density, the consistency coefficient, the fluid index and the material constants of the fluid are $\rho, k, n$ and $A_{1}, n_{1}$, respectively, the average flow rate and the pressure gradient of the fluid in the annulus are $Q$ and $P$, respectively, and temperature of the fluid is $t$.

\section{B. Equation of motion}

The equation of motion of the unsteady flow of the second-order fluid in eccentric annulus with the inner cylinder reciprocating axially under the bipolar coordinate system $(\xi, \zeta, z)$ is

$$
\begin{aligned}
\rho \frac{\partial w}{\partial t}= & P+\frac{c^{2}+s^{2}}{C^{2}}\left\{\frac{\partial}{\partial \xi}\left(\eta \frac{\partial w}{\partial \xi}\right)+\frac{\partial}{\partial \zeta}\left(\eta \frac{\partial w}{\partial \zeta}\right)\right. \\
& \left.-\frac{1}{2}\left[\frac{\partial}{\partial \xi}\left(\theta_{1} \frac{\partial^{2} w}{\partial \xi \partial t}\right)+\frac{\partial}{\partial \zeta}\left(\theta_{1} \frac{\partial^{2} w}{\partial \zeta \partial t}\right)\right]\right\},
\end{aligned}
$$

where $w$ is axial fluid velocity, the expressions of $c$ 、 $s 、 C 、 \eta 、 \theta_{1}$ and $I_{2}$ can be referred to [4].

\section{Initial and boundary conditions}

The initial condition is

$$
w(\xi, \zeta, 0)=w_{\mathrm{P}}(\xi, \zeta),
$$

where $w_{\mathrm{P}}(\xi, \zeta)$ is the velocity distribution function of the Poiseuille flow of the second-order fluid in eccentric annulus with the inner cylinder static under the bipolar coordinate system, which can be obtained through numerical method.

The boundary conditions are

$$
\begin{gathered}
w\left(\xi_{\text {in }}, \zeta, t\right)=U(t), \\
w\left(\xi_{\text {out }}, \zeta, t\right)=0, \\
\frac{\partial w(\xi, 0, t)}{\partial \zeta}=\frac{\partial w(\xi, \pi, t)}{\partial \zeta}=0 .
\end{gathered}
$$

\section{NUMERICAL METHOD FOR SOLVING THE GOVERNING EQUATIONS}

\section{A. Discretion of the governing equations}

$D=\left\{(\xi, \zeta) \mid \xi_{\text {in }} \leq \xi \leq \xi_{\text {out }}, 0 \leq \zeta \leq \pi\right\} \quad$, the rectangular compu- ting area is sectioned into equidistance grids, where $\xi_{\text {in }}$ corresponds to the outer wall of the inner cylinder and $\xi_{\text {out }}$ to the inner wall of the outer cylinder; $\zeta=0$ corresponds to the widest clearance of the annulus and $\zeta=\pi$ to the narrowest clearance; $h_{1}$ and $h_{2}$ are the steps in $\xi$ and $\zeta$ directions, respectively, shown in Figure 1.

The volume of the control volume $Z$ under the bipolar coordinate system is given as

$$
\Delta V=h_{\xi} h_{\zeta} h_{1} h_{2}
$$

where $h_{\xi}$ and $h_{\zeta}$ are the Lame coefficients under the bipolar coordinate system.

Take an integral on (1) from $t$ to $t+\Delta t$ on the control volume $Z$, with Fully Implicit scheme taken on time integral. Let $w^{l}$ and $w^{l+1}$ express the velocity values at $t$ and $t+\Delta t$, respectively, and connected with (6), we can obtain

$$
a_{Z} w_{Z}^{k+1}+a_{N} w_{N}^{k+1}+a_{S} w_{S}^{k+1}+a_{E} w_{E}^{k+1}+a_{W} w_{W}^{k+1}=b,
$$

where the nodes $Z, E, W, S$ and $N$ correspond with $(i, j),(i+1, j),(i-1, j),(i, j+1),(i, j-1)$, respectively, as shown in Figure 1.

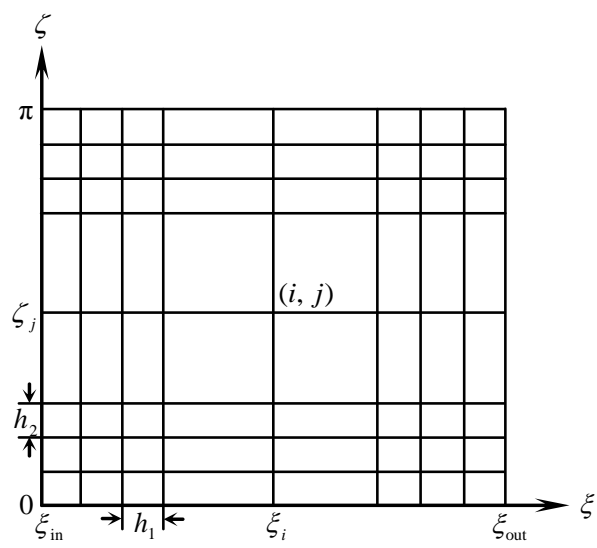

Fig 1. Subdivision of grids of calculation area

\section{B. Numerical method and process}

Through arraying the coefficients of (7) by the natural sequence method, a five diagonal matrix can be obtained. Here, ADI method is adopted to solve the discrete equation group, which can guarantee unconditional stability during the solving process and is easy to get the solution under a certain accuracy requirement, and in addition, has advantages of less memory occupation and faster computing speed.

Within an arbitrary time step $h_{3}$, namely, the process of time layer $k \rightarrow k+1$, divide an arbitrary iteration process $l \rightarrow l+1$ into two steps. In the former step $l \rightarrow l+1 / 2$, in $\xi$ direction, $w_{Z}^{k+1}, w_{E}^{k+1}$ and $w_{W}^{k+1}$ are expressed as $w_{Z}^{l+1 / 2, k+1}, w_{E}^{l+1 / 2, k+1}$ and $w_{W}^{l+1 / 2, k+1}$, respectively, in which the velocity value $w^{l+1 / 2, k+1}$ is regarded to be unknown, while in $\zeta$ direction, $w_{N}^{k+1}$ and $w_{S}^{k+1}$ are expressed as $w_{N}^{l, k+1}$ and $w_{S}^{l, k+1}$, respectively, in which $w^{l, k+1}$ is the velocity value obtained after the last iteration and is regarded to be known. So, (7) can be changed to

$$
\begin{aligned}
& a_{Z}^{l, k+1} w_{Z}^{l+1 / 2, k+1}+a_{E}^{l, k+1} w_{E}^{l+1 / 2, k+1}+a_{E}^{l, k+1} w_{W}^{l+1 / 2, k+1} \\
& =b^{l, k+1}-\left(a_{N}^{l, k+1} w_{N}^{l, k+1}+a_{S}^{l, k+1} w_{S}^{l, k+1}\right)
\end{aligned}
$$

Therefore, (8) is the iteration formula scanning in $\xi$ direction.

Particularly, when initially iterating at present time layer, the iteration formula in $\xi$ direction is

$$
a_{Z}^{k} w_{Z}^{l+1 / 2, k+1}+a_{E}^{k} w_{E}^{l+1 / 2, k+1}+a_{E}^{k} w_{W}^{l+1 / 2, k+1}=b^{k}-\left(a_{N}^{k} w_{N}^{k}+a_{S}^{k} w_{S}^{k}\right)
$$

(8) and (9) are both tridiagonal equation groups, which can be solved using Thomas method, so that $w^{l+1 / 2, k+1}$ of each node on line $j$ can be obtained, and so does 
$w^{l+1 / 2, k+1}$ of each node in the whole grid area, scanned line by line like this.

Then, in the latter step $l+1 / 2 \rightarrow l+1$, in $\zeta$ direction, $w_{Z}^{k+1}, w_{N}^{k+1}$ and $w_{S}^{k+1}$ are expressed as $w_{Z}^{l+1, k+1}, w_{N}^{l+1, k+1}$ and $w_{S}^{l+1, k+1}$, respectively, in which the velocity value $w^{l+1, k+1}$ is regarded to be unknown, while in $\xi$ direction, $w_{E}^{k+1}$ and $w_{W}^{k+1}$ are expressed as $w_{N}^{l+1 / 2, k+1}$ and $w_{S}^{l+1 / 2, k+1}$, respectively, in which $w^{l+1 / 2, k+1}$ is the velocity value obtained after the iteration in former semi-step and is regarded to be known. So, (7) can be changed to

$$
\begin{aligned}
& a_{Z}^{l+1 / 2, k+1} w_{Z}^{l+1, k+1}+a_{E}^{l+1 / 2, k+1} w_{E}^{l+1, k+1}+a_{W}^{l+1 / 2, k+1} w_{W}^{l+1, k+1} \\
= & b^{l+1 / 2, k+1}-\left(a_{N}^{l+1 / 2, k+1} w_{N}^{l+1 / 2, k+1}+a_{S}^{l+1 / 2, k+1} w_{S}^{l+1 / 2, k+1}\right) .
\end{aligned}
$$

So, (10) is the iteration formula scanning in $\zeta$ direction.

Equation (10) is a tridiagonal equation group as well, which can be solved using Thomas method again, so that $w^{l+1, k+1}$ of each node on row $i$ can be obtained, and so does $w^{l+1, k+1}$, the velocity value after one iteration at present time layer, of each node in the whole grid area, scanned row by row like this.

The convergence criterion is given as

$$
\left|\frac{w^{l+1, k+1}-w^{l, k+1}}{w^{l+1, k+1}}\right| \leq \varepsilon,
$$

where $\varepsilon$ is convergence accuracy, here $\varepsilon=10^{-6}$.

After the two alternating direction scanning process mentioned above, the solutions of the discrete equation group can be obtained at time $k+1$, and by this way, the solutions at each time can be obtained in a sequent manner and the velocity field $w(\xi, \zeta, t)$ is obtained finally.

\section{PRESSURE GRADIENT EQUATION}

Equation (1) can be written as

$$
\begin{aligned}
& P=\rho \frac{\partial w}{\partial t}-\frac{c^{2}+s^{2}}{C^{2}}\left\{\frac{\partial}{\partial \xi}\left(\eta \frac{\partial w}{\partial \xi}\right)+\frac{\partial}{\partial \zeta}\left(\eta \frac{\partial w}{\partial \zeta}\right)\right. \\
& \left.-\frac{1}{2}\left[\frac{\partial}{\partial \xi}\left(\theta_{1} \frac{\partial^{2} w}{\partial \xi \partial t}\right)+\frac{\partial}{\partial \zeta}\left(\theta_{1} \frac{\partial^{2} w}{\partial \zeta \partial t}\right)\right]\right\}=p(w),
\end{aligned}
$$

SO,

$$
w=p^{-1}(P) .
$$

From (13), it can be seen that there is some functional relationship between $w$ and $P$. From the formula of flow rate of the unsteady flow of the second-order fluid in eccentric annulus with the inner cylinder reciprocating axially in a period $T$, the following is obtained:

$$
Q=\frac{1}{T} \int_{0}^{T} \int_{0}^{2 \pi} \int_{\xi_{m}}^{\xi_{m}} \frac{C^{2}}{c^{2}+s^{2}} w \mathrm{~d} \xi \mathrm{d} \zeta \mathrm{d} t=q(w) .
$$

By substituting (13) into (14), the following equation is obtained:

$$
Q=q\left[p^{-1}(P)\right],
$$

Let $f(P)=q\left[p^{-1}(P)\right]$, then

$$
Q=f(P) \text {. }
$$

If $P_{\mathrm{k}}$ is any pressure gradient, and $Q_{\mathrm{k}}$ is its corresponding average flow rate, then from (16), the following is obtained:

$$
Q_{\mathrm{k}}=f\left(P_{\mathrm{k}}\right),
$$

Let $F=Q_{\mathrm{k}}-Q$. When $F=0$, the following is obtained:

$$
Q_{\mathrm{k}}-Q=0 \text {. }
$$

Therefore, (18) is the pressure gradient equation of unsteady flow of the second-order fluid with variable coefficients in eccentric annulus with the inner cylinder reciprocating axially under the condition of a given average flow rate, which can only solved by using numerical calculation method.

\section{EXPERIMENT}

The experimental apparatus and flow schematic diagram is the same as in [7]. $R_{\text {out }}=0.035 \mathrm{~m}, R_{\text {in }}=0.010 \mathrm{~m}$. HPAM aqueous solution, the mass fraction concentrations of which is $0.1 \%$, regarded as the second-order fluid with variable coefficient, was used as experimental fluid. The rheological parameters, measured by a HAAKE-RS150 rheometer, are shown in TABLE 1.

TABLE 1 Rheological data of $0.1 \%$ HPAM aqueous solution

\begin{tabular}{|c|c|c|c|c|c|}
\hline $\begin{array}{c}t \\
\left({ }^{\circ} \mathrm{C}\right)\end{array}$ & $\begin{array}{c}\rho \\
\left(\mathrm{kg} \cdot \mathrm{m}^{-3}\right)\end{array}$ & $\begin{array}{c}k \\
\left(\mathrm{~Pa} \cdot \mathrm{s}^{n}\right)\end{array}$ & $n$ & $A_{1}$ & $n_{1}$ \\
\hline 14.9 & 999.1 & 0.1117 & 0.7408 & 1.7822 & -0.4569 \\
\hline
\end{tabular}

Under the same conditions, when $e p=0.48, S=1.0 \mathrm{~m}$, $f=0.167 \mathrm{~Hz}$, on the basis of numerically solving the governing equations, using FDM-CN-GS, FVM-CN-SOR and FVM-FI-ADI method, respectively, to obtain the velocity field, connected with the pressure gradient equation (18), the coincidences between numerically calculated pressure gradients and the experimentally measured ones are compared, shown in TABLE 2, where the experimentally measured pressure gradients are all taken from the laminar zones. The discretion steps are $h_{1}=\pi / 40, h_{2}=\left(\xi_{\text {out }}-\xi_{\text {in }}\right) / 20, h_{3}=T / 20$.

TABLE 2 Numerical calculation of the flow of

\begin{tabular}{|c|c|c|c|c|c|c|c|}
\hline $\begin{array}{c}\mathrm{Q} \\
\left(10^{-4} \mathrm{~m}^{3} \cdot \mathrm{s}^{-1}\right)\end{array}$ & $\begin{array}{c}P_{1} \\
\left(\mathrm{~Pa} \cdot \mathrm{m}^{-1}\right)\end{array}$ & $\begin{array}{c}P_{2} \\
\left(\mathrm{~Pa} \cdot \mathrm{m}^{-1}\right)\end{array}$ & $\begin{array}{c}P_{3} \\
\left(\mathrm{~Pa} \cdot \mathrm{m}^{-1}\right)\end{array}$ & $\begin{array}{c}\mathrm{P}^{\cdot} \\
\left(\mathrm{Pa} \cdot \mathrm{m}^{-1}\right)\end{array}$ & $\begin{array}{c}\triangle_{1} \\
(\%)\end{array}$ & $\begin{array}{c}\triangle_{2} \\
(\%)\end{array}$ & $\begin{array}{c}\triangle_{3} \\
(\%)\end{array}$ \\
\hline 0.550 & 20.64 & 20.62 & 20.54 & 19.66 & -4.98 & -4.88 & -4.48 \\
\hline 0.925 & 27.93 & 27.90 & 27.84 & 26.03 & -7.30 & -7.18 & -6.95 \\
\hline 1.224 & 33.95 & 33.89 & 33.46 & 32.41 & -4.75 & -4.57 & -3.24 \\
\hline 1.669 & 40.98 & 40.87 & 40.55 & 39.38 & -4.06 & -3.78 & -2.97 \\
\hline 2.013 & 46.38 & 46.21 & 45.34 & 44.98 & -3.11 & -2.73 & -0.80 \\
\hline 2.142 & 48.09 & 48.38 & 47.05 & 46.32 & -3.82 & -4.45 & -1.58 \\
\hline 2.465 & 52.18 & 52.46 & 51.28 & 51.02 & -2.27 & -2.82 & -0.51 \\
\hline 2.753 & 54.56 & 54.88 & 54.92 & 55.41 & 1.53 & 0.96 & 0.88 \\
\hline 3.009 & 56.02 & 56.79 & 58.27 & 58.87 & 4.84 & 3.53 & 1.02 \\
\hline $\bar{\Delta}(\%)$ & & & & & 4.08 & 3.88 & 2.49 \\
\hline
\end{tabular}

In TABLE 2, $P_{1}, P_{2}$ and $P_{3}$ are the pressure gradients calculated using FDM-CN-GS, FVM-CN-LSOR and FVM-FI-ADI method, respectively; $\Delta_{1}, \Delta_{2}$ and $\Delta_{3}$ are 
corresponding percentage errors of pressure gradients, $\Delta_{i}=\left(P^{*}-P_{i}\right) / P^{*} \times 100 \%, i=1,2,3 ; \bar{\Delta}$ is average relative error of pressure gradient, $\bar{\Delta}=\frac{1}{n} \sum_{j=1}^{n}\left|\Delta_{i j}\right|$.

From TABLE 2, it can be seen that under the same conditions, the average relative error of pressure gradient calculated using FVM-FI-ADI, the new method presented, is smaller than that using FDM-CN-GS or FVM-CN-SOR method, which means the coincidences between the numerically calculated pressure gradients and the experimentally measured ones using FVM-FI-ADI method are better than those using the other two methods. Moreover, the numerical calculating procedure of FVMFI-ADI method is much more simple and convenient and the calculating speed is faster.

\section{CONCLUSIONS}

(1) FVM-FI-ADI method is presented to numerically solve the governing equations of unsteady flow of the second-order fluid with variable coefficients in eccentric annulus with the inner cylinder reciprocating axially under the bipolar coordinate system, in which FVM is used to discrete the governing equations, in which FI scheme is taken on time integral, and ADI method to solve the discrete equation group.

(2) Using $0.1 \%$ HPAM aqueous solution, under the same conditions, the experimental results show that the new method is testified to be correct and the coincidences between the calculated pressure gradients and the measured ones using the new method are better than those using the old methods; moreover, the numerical calculating procedure of the new method is much more simple and convenient and the calculating speed is faster.

\section{REFERENCES}

[1] Noriyasu Mori, Takaaki Eguchi, Kiyoji Nakamura, "Pressure flow of non-Newtonian fluids between eccentric double cylinders with the inner cylinder rotating. Part1: Numerical calculation", Journal of the Textile Machinery Society of Japan, vol. 2, Apr. 1987, pp. 46-53.

[2] Noriyasu Mori, Mitsuhiro Yagami, Takaaki Eguchi, "Pressure flow of non-Newtonian fluids between eccentric double cylinders with the inner cylinder rotating. Part2: Experiment", Journal of the Textile Machinery Society of Japan, vol. 3, Jun. 1987, pp. 73-77.

[3] Y. J. Yang, "Unsteady flow of viscoelastic fluid in eccentric annuli with inner cylinder reciprocating axially", Daqing: Doctor Dissertation of Daqing Petroleum Institute, 2005, pp. 28-42.

[4] Y. Wang, H. Q. Cui, Y. J. Yang, J. H. Guo, N. Li, "Pressure distribution on the wall of the inner cylinder reciprocating axially to the unsteady flow of viscoelastic fluid in eccentric annulus", Journal of Hydro- dynamics, Ser. B, vol. 5, Oct. 2006, pp. 606-612.

[5] X. N. Zhang, "Numerical calculation of flow rate of swabbed fluid flowing in wellbore under polymer-driving operating mode", Daqing: Master Dissertation of Daqing Petroleum Institute, 2007, pp. 28-35.

[6] H. Q. Cui, X. N. Zhang, N. Li, "Flow rate distribution of the nonsteady flow of second-order fluid in eccentric annulus with inner cylinder reciprocating axially", Journal of Daqing Petroleum Institute, vol. 3, Jun. 2007, pp. 21-24.

[7] N. Li, X. L. Song, X. N. Zhang, "Numerical calculation of average flow rate of the unsteady flow of a viscoelastic fluid in eccentric annulus with the inner cylinder reciprocating axially", Journal of Daqing Petroleum Institute, vol. 2, Apr. 2009, pp. 44-47.

[8] Y. Chang, Z. J. Bao, H. Q. Cui, N. Li, "Pressure gradient of unteady flow of fluid in eccentric annulus with inner cylinder reciprocating axially", Journal of Daqing Petroleum Institute, vol. 6, Dec. 2007, pp. 40-43.

[9] S. R. Yang, "Numerical calculation of Unsteady flow of viscoelastic fluid in eccentric annulus", Daqing: Doctor Dissertation of Daqing Petroleum Institute, 2006, pp. 35-37.

[10] Patankar, "Numerical calculation of heat transfer and fluid flow", Beijing: Science Press, 1989, pp. 1-20. 